Relations industrielles

Industrial Relations

\title{
Côté, Nicole, Laurent Bélanger et Jocelyn Jacques, $L a$ dimension humaine des organisations
}

\section{Gérard Ouimet}

Volume 50, numéro 2, 1995

URI : https://id.erudit.org/iderudit/051020ar

DOI : https://doi.org/10.7202/051020ar

Aller au sommaire du numéro

Éditeur(s)

Département des relations industrielles de l'Université Laval

ISSN

0034-379X (imprimé)

1703-8138 (numérique)

Découvrir la revue

Citer ce compte rendu

Ouimet, G. (1995). Compte rendu de [Côté, Nicole, Laurent Bélanger et Jocelyn Jacques, La dimension humaine des organisations]. Relations industrielles /

Industrial Relations, 50(2), 448-449. https://doi.org/10.7202/051020ar

Tous droits réservés @ C Département des relations industrielles de l'Universite Laval, 1995
Ce document est protégé par la loi sur le droit d'auteur. L’utilisation des services d'Érudit (y compris la reproduction) est assujettie à sa politique d'utilisation que vous pouvez consulter en ligne.

https://apropos.erudit.org/fr/usagers/politique-dutilisation/ 


\section{Recensions}

\section{Book Reviews}

\section{La dimension humaine des organisations}

par Nicole CÔTÉ, Laurent BÉLANGER et Jocelyn JACQUES, Boucherville, Québec, Gaëtan Morin éditeur, 1994, 396 p., ISBN 2-89105-480-6.

D'entrée de jeu, il convient de reconnaître le caractère essentiellement général de l'ouvrage. Il s'agit d'un livre de base portant sur le comportement organisationnel. Les auteurs se fixent pour objectif " de fournir à ceux et celles qui s'intéressent au monde organisationnel un condensé des concepts et des recherches les plus pertinentes à la compréhension du comportement et du vẻcu des personnes dans les milieux de travail ". La lecture attentive de l'ouvrage nous permet d'affirmer que les auteurs ont atteint en partie seulement leur objectif.

Certes, l'ouvrage La dimension humaine des organisations est un bon condensé de toute une littérature analysant la dynamique du comportement humain au travail. L'écriture y est limpide, les sections bien découpées et la compréhension de la matière soumise à l'étude est facilement accessible aux néophytes. C'est assurément sa plus grande qualité. Toutefois, nonobstant la simplicité et la clarté des développements analytiques, la facture de l'ouvrage est décevante à cause de son manque d'originalité. Hormis certains timides passages faisant référence à la réalité sociétale et organisationnelle québécoise, le livre pourrait volontiers passer pour une traduction d'un ouvrage américain. À l'instar d'une myriade de volumes pédagogiques destinés à l'enseignement collégial et universitaire de tout premier niveau, ce livre aborde de façon classique les traditionnelles thématiques affé- rentes à ce champ disciplinaire, telles la personnalité, la perception, les valeurs, la motivation, les groupes et leur dynamique, le leadership, le pouvoir, la communication, et le changement.

Mais, et c'est là où les choses commencent vraiment à se gâter, il n'y a pas que le choix des thématiques qui soit traditionnel : leur traitement analytique respecte scrupuleusement une vision des choses figée, depuis déjà plusieurs décennies, dans un paradigme foncièrement fonctionnaliste. C'est ainsi qu'à chacun des chapitres - ou presque -, nous avons droit, dans un premier temps, à une compendieuse description des différentes écoles de pensée traitant de la thématique à l'étude, pour finalement aboutir à une fort brève conclusion reprenant ici et là quelques éléments saillants du développement précédent. L'analyse ne dépasse jamais le niveau primaire de la description. $\mathrm{Pa}$ reille superficialité analytique prête flanc à deux critiques majeures.

Tout d'abord, l'ouvrage constitue en fait la troisième mouture de réflexions entreprises il y a déjà plusieurs années - Les aspects humains de l'organisation (1979) et Individu, groupe et organisation (1986). Or, cette troisième mouture (La dimension humaine des organisations, 1994) est à peu de choses près identique aux deux précédentes. Bien sūr, les auteurs se sont efforcés de rafraîchir leurs écrits en y greffant quelques ajouts bibliographiques, mais 
l'infrastructure analytique demeure vieille. Tout au long des 396 pages de l'ouvrage, il s'avère excessivement difficile de glaner quelque chose de nouveau. L'anachronisme de l'ouvrage est à ce point criant que les conclusions brossées par les auteurs rejoignent au mieux, sans jamais les dépasser, celles d'une ribambelle de manuels scolaires américains publiés vingt ans auparavant. Qui plus est, le désagréable "sentiment de déjà vu " s'exacerbe davantage au contact d'ouvrages récents ayant poussé plus loin la réflexion de la dynamique individu-organisation. Par exemple, le collectif publié sous la direction de JeanFrançois Chanlat et intitulé L'individu dans l'organisation: les dimensions oubliées (1990) permet très bien de saisir l'obligation de sortir définitivement des sentiers battus afin d'accéder à une connaissance plus perspicace de la complexité de l'humain et du social. C'est ainsi qu'un regard sur les dimensions psychique, symbolique et spatio-temporelle aurait indubitablement permis une appréhension plus fine, subtile, congrue et complète de la vie des individus au travail.

Puis, l'ouvrage souffre d'un manque de synthèse. Il n'y a pas, ou trop peu, de réflexions critiques permettant d'extraire, des diverses descriptions syncrétiques, un fil conducteur liant entre elles les diverses notions théoriques abordées. Le manque de capsulage analytique mine considérablement tant la profondeur des réflexions que la portée des conclusions. En outre, la ténuité de l'exercice critique opéré par les auteurs se solde en définitive par la présentation " acontextuelle" des diverses constructions théoriques. À cet égard, les deux chapitres traitant de la motivation au travail (chapitres 7 et 8 ) détonnent étonnamment avec l'actuelle réalité sociétale et organisationnelle. Peut-on encore parler de motivation instrumentale (Maslow, Herzberg et Alderfer) dans un cadre organisationnel postmoderne où l'animation du psychisme (voir les travaux de Pagès, Enriquez, Lipovetsky, Ehrenberg et compagnie) revêt désormais un rôle incontournable? Peut-on encore aborder l'étude de la mobilisation instrumentale (approche socio-technique, QVT et qualité totale) dans une période de désengagement systématique des entreprises à l'endroit de leurs employés? En somme, parce qu'ils appartiennent à une autre époque, bon nombre des instruments conceptuels recensés et présentés par les auteurs ne parviennent plus à rendre compte judicieusement de la dynamique des comportements humains émis dans des organisations de plus en plus réticulaires, aux prises avec un éclatement des marchés. À l'évidence, il apparaît nettement que l'approche analytique fonctionnaliste des auteurs convient davantage aux réalités des années 70 qu'à celles d'aujourd'hui.

\section{GÉRARD OUIMET École des Hautes Études Commerciales}

\section{The Changing Workplace: Reshaping Canada's Industrial Relations System by Daniel DRACHE and Harry GLASBEEK, Toronto, James Lorimer \& Company, Publishers, 1992, 306 p., ISBN 1-55028-405-3 (Bond) and ISBN 1-55028-404-5 (PBK).}

Drache and Glasbeek, writing from an unapologetically pro-labour perspective, put forward their proposals for changing both the Canadian industrial relations system and current macroeconomic policy in their book
The Changing Workplace, appropriately subtitled Reshaping Canada's Industrial Relations System. This work is not primarily a study about how Canada's workplaces have changed over the past several decades; rather, their approach 